From the Laboratory of Molecular Genetics, The Oncogenomics Center, Institute for Cancer Research and Treatment, University of Torino Medical School, Candiolo; The Falck Division of Medical Oncology, Ospedale Niguarda Ca' Granda; Italian Foundation for Cancer Research-Institute of Molecular Oncology, Milan, Italy; Laboratory of Molecular Diagnostic, Istituto Cantonale di Patologia, Locarno; and Oncology Institute of Southern Switzerland, Ospedale San Giovanni, Bellinzona, Switzerland

Submitted June 3, 2008; accepted August 28, 2008; published online ahead of print at www.jco.org on November 10, 2008

Supported by the Italian Association for Cancer Research, Italian Ministry of Health, Regione Piemonte, Italian Ministry of University and Research, Association for International Cancer Research, EU FP6 MCSCs Contract No. 037297, CRT Progetto Alfieri, Oncosuisse Grant No. OCS-01921-08-2006, Fondazione Ticinese per la Ricerca sul Cancro, and Oncologia Ca' Granda Onlus Fondazione.

F.D.N., M.M., and F.M. contributed equally to this work.

M.F., S.S., and A.B. are co-senior authors.

Terms in blue are defined in the glossary, found at the end of this article and online at www.jco.org

Authors' disclosures of potential conflicts of interest and author contributions are found at the end of this article.

Corresponding author: Alberto Bardelli, PhD, Laboratory of Molecular Genetics, The Oncogenomics Center, Institute for Cancer Research and Treatment, University of Torino, Medical School, Str prov $142 \mathrm{Km} 3.95,10060$ Candiolo, Torino, Italy; e-mail: a.bardelli@unito.it.

The Appendix is included in the full-text version of this article, available online at www.jco.org. It is not included in the PDF version (via Adobe $®$ Reader $\circledast$ )

(C) 2008 by American Society of Clinical Oncology

$0732-183 \times / 08 / 2635-5705 / \$ 20.00$

DOI: 10.1200/JCO.2008.18.0786

\title{
Wild-Type BRAF Is Required for Response to Panitumumab or Cetuximab in Metastatic Colorectal Cancer
}

Federica Di Nicolantonio, Miriam Martini, Francesca Molinari, Andrea Sartore-Bianchi, Sabrina Arena, Piercarlo Saletti, Sara De Dosso, Luca Mazzucchelli, Milo Frattini, Salvatore Siena, and Alberto Bardelli

\section{$\begin{array}{lllllllll}\text { A } & \text { B } & \text { S } & \text { T } & \text { R } & \text { A } & \text { C } & T\end{array}$}

\section{Purpose}

Cetuximab or panitumumab are effective in $10 \%$ to $20 \%$ unselected metastatic colorectal cancer (CRC) patients. KRAS mutations account for approximately $30 \%$ to $40 \%$ patients who are not responsive. The serine-threonine kinase BRAF is the principal effector of KRAS. We hypothesized that, in KRAS wild-type patients, BRAF mutations could have a predictive/prognostic value.

\section{Patients and Methods}

We retrospectively analyzed objective tumor responses, time to progression, overall survival (OS), and the mutational status of KRAS and BRAF in 113 tumors from cetuximab- or panitumumabtreated metastatic CRC patients. The effect of the BRAF V600E mutation on cetuximab or panitumumab response was also assessed using cellular models of CRC.

\section{Results}

KRAS mutations were present in $30 \%$ of the patients and were associated with resistance to cetuximab or panitumumab $(P=.011)$. The BRAF V600E mutation was detected in 11 of 79 patients who had wild-type KRAS. None of the BRAF-mutated patients responded to treatment, whereas none of the responders carried $B R A F$ mutations $(P=.029)$. BRAF-mutated patients had significantly shorter progression-free survival $(P=.011)$ and $O S(P<.0001)$ than wild-type patients. In CRC cells, the introduction of BRAF V600E allele impaired the therapeutic effect of cetuximab or panitumumab. Treatment with the BRAF inhibitor sorafenib restored sensitivity to panitumumab or cetuximab of CRC cells carrying the V600E allele.

\section{Conclusion}

$B R A F$ wild-type is required for response to panitumumab or cetuximab and could be used to select patients who are eligible for the treatment. Double-hit therapies aimed at simultaneous inhibition of epidermal growth factor receptor and BRAF warrant exploration in CRC patients carrying the V600E oncogenic mutation.

\section{J Clin Oncol 26:5705-5712. (C) 2008 by American Society of Clinical Oncology}

\section{INTRODUCTION}

Patients with metastatic colorectal cancer (mCRC) have a 5-year survival rate of less than $10 \% .{ }^{1}$ Cetuximab and panitumumab, two monoclonal antibodies (MoAb) targeting epidermal growth factor receptor (EGFR), have recently entered clinical practice and have proven to be effective in providing clinical benefit in approximately $10 \%$ to $20 \%$ of patients. ${ }^{2-5}$ Both molecules bind to the extracellular domain of EGFR, thus leading to inhibition of its downstream signaling. EGFR is a transmembrane tyrosine kinase receptor that, on ligand binding, triggers two main signaling pathways. These include the RAS-RAF-MAPK axis, which is mainly involved in cell proliferation, and the PI3KPTEN-AKT pathway, which is mainly involved in cell survival and motility. ${ }^{6}$ Cetuximab and panitu- mumab are registered for mCRC patients whose tumors express EGFR protein as determined by immunohistochemistry. However, it has been clearly demonstrated that this method is not predictive of treatment efficacy. ${ }^{2,3,7}$ On the contrary, recent data indicate that EGFR gene status evaluated by fluorescent in situ hybridization or chromogenic in situ hybridization may predict response to these MoAbs. ${ }^{8-12}$ We and others have previously shown, at the preclinical ${ }^{13}$ and clinical ${ }^{8-10,14-18}$ level, that the presence of mutated KRAS alleles is an independent predictive marker of anti-EGFR MoAb resistance. On the basis of these results, the European Union drug regulatory body, the European Medicines Agency, has approved the use of panitumumab only for mCRC patients whose tumors display wild-type KRAS. However, the occurrence of KRAS mutations only accounts for approximately $30 \%$ to $40 \%$ of 
nonresponsive patients. ${ }^{8-10,14,15}$ Therefore, the identification of additional genetic determinants of primary resistance to EGFR-targeted therapies in colorectal cancers (CRCs) is important at least for two reasons. The first is to prospectively identify patients who should not receive either cetuximab or panitumumab, thus avoiding their exposure to ineffective and expensive therapy. Second, understanding the molecular basis of primary resistance to EGFR-targeted MoAb therapies could allow the rationale design of alternative treatment strategies. We hypothesized that, in the absence of KRAS mutations, resistance to anti-EGFR treatments could be caused by alterations of other members of the RAS-RAF-MAPK pathway. Using genetic analysis of mCRCs from cetuximab- or panitumumab-treated patients and cellular models of CRC, we assessed whether BRAF mutations affect the response to therapies based on EGFR-targeted MoAbs. Because BRAF mutations are linked to microsatellite instability (MSI), a condition generally associated with better prognosis and resistance to standard chemotherapy, ${ }^{19}$ we also investigated MSI in our cohort of treated patients.

\section{PATIENTS AND METHODS}

\section{Patient Population and Treatment Regimens}

We retrospectively analyzed 113 patients with histologically confirmed mCRC either at Ospedale Niguarda Ca' Granda (Milan, Italy) or at the Institute of Pathology (Locarno, Switzerland). Patients evaluated in this study were selected based on evidence that treatment outcome could be attributable only to administration of either panitumumab or cetuximab. Patients were enrolled onto clinical trials ( 64 of 113 patients; see Appendix, online only) ) $^{3,5,20-23}$ or received panitumumab or cetuximab as per label indication. For patients who experienced progression on irinotecan-based chemotherapy, cetuximab was administered in combination with irinotecan at the same dose and schedule previously used. In all patients, refractoriness to irinotecan was defined as documented disease progression by comparison of computed tomography or magnetic resonance imaging scans during or within 3 months of receiving an irinotecan regimen (administered for at least 6 weeks). Besides the earlier mentioned inclusion criteria, the availability of a tumor sample qualitatively and quantitatively suitable for molecular analyses was also a requirement for being considered in the present study. All patients had EGFR expression in their tumor specimens in $\geq 1 \%$ of malignant cells assessed by immunohistochemistry with the Dako EGFR PharmDx kit (DakoCytomation, Glostrup, Denmark). Patients' baseline characteristics, disease stage, and number of previous lines of therapy are listed in Table 1 . Treatment was continued until progressive disease (PD) or toxicity occurred, according to the standard criteria. ${ }^{24}$

\section{Clinical Evaluation and Tumor Response Criteria}

Clinical response was assessed every 6 to 8 weeks with radiologic examination (computed tomography or magnetic resonance imaging). The Response Evaluation Criteria in Solid Tumors ${ }^{24}$ were adopted for evaluation, and objective tumor response was classified as partial response (PR), stable disease (SD), or PD. Patients with SD or PD were defined as nonresponders. ${ }^{24}$ Two independent oncologists and radiologists verified, in a blinded manner, the clinical response for all patients.

\section{Molecular Analyses}

Formalin-fixed, paraffin-embedded tumor blocks were reviewed for quality and tumor content, and a single representative tumor block from each patient, containing at least $70 \%$ of neoplastic cells, was selected. Genomic DNA was extracted as previously described. ${ }^{8}$

\section{MSI}

The status of MSI was assessed by the analysis of the microsatellite loci included in the panel of Bethesda (BAT25, BAT26, D2S123, D5S346, and D17S250), as previously reported. ${ }^{25}$ MSI was confirmed by the presence of an additional peak in tumor sample compared with normal paired tissue. MSI was defined as being present when more than $30 \%$ of loci showed instability.

\section{Mutational Analysis of KRAS and BRAF in Tumor Samples}

We searched for KRAS and BRAF point mutations in exons 2 and 15, respectively. These two exons include codons 12 and 13 of KRAS and codon

\begin{tabular}{|c|c|c|c|c|c|c|c|c|}
\hline \multirow[b]{2}{*}{ Characteristic } & \multicolumn{2}{|c|}{ Mutant KRAS } & \multicolumn{2}{|c|}{ Mutant BRAF } & \multicolumn{2}{|c|}{$\begin{array}{c}\text { Wild-Type KRAS and } \\
B R A F\end{array}$} & \multicolumn{2}{|l|}{ Total } \\
\hline & No. of Patients & $\%$ & No. of Patients & $\%$ & No. of Patients & $\%$ & No. of Patients & $\%$ \\
\hline No. of patients & 34 & 30 & 11 & 10 & 68 & 60 & 113 & 100 \\
\hline Male sex & 16 & 14 & 8 & 7 & 56 & 50 & 80 & 71 \\
\hline \multicolumn{9}{|l|}{ Baseline age, years } \\
\hline Median & \multicolumn{2}{|l|}{64} & \multicolumn{2}{|l|}{63} & \multicolumn{2}{|l|}{62} & \multicolumn{2}{|l|}{63} \\
\hline Range & \multicolumn{2}{|l|}{$48-82$} & \multicolumn{2}{|l|}{$43-68$} & \multicolumn{2}{|l|}{$26-85$} & \multicolumn{2}{|l|}{$26-85$} \\
\hline \multicolumn{9}{|l|}{ Site of primary disease } \\
\hline Colon & 21 & 19 & 10 & 9 & 39 & 35 & 70 & 62 \\
\hline Rectum & 13 & 12 & 1 & 1 & 29 & 26 & 43 & 38 \\
\hline \multicolumn{9}{|l|}{ Anti-EGFR MoAb } \\
\hline Cetuximab & 12 & 11 & 3 & 3 & 21 & 19 & 36 & 32 \\
\hline Cetuximab plus chemotherapy & 12 & 11 & 2 & 2 & 37 & 33 & 51 & 45 \\
\hline Panitumumab & 10 & 9 & 6 & 5 & 10 & 9 & 26 & 23 \\
\hline Received prior adjuvant chemotherapy* & 31 & 27 & 10 & 9 & 59 & 52 & 100 & 88 \\
\hline \multicolumn{9}{|l|}{ No. of prior lines of chemotherapy } \\
\hline 1 & 4 & 4 & 2 & 2 & 6 & 5 & 12 & 11 \\
\hline 2 & 14 & 12 & 4 & 4 & 35 & 31 & 53 & 47 \\
\hline 3 & 11 & 10 & 3 & 3 & 15 & 13 & 29 & 26 \\
\hline 4 & 2 & 2 & 1 & 1 & 2 & 2 & 5 & 4 \\
\hline 5 & 0 & 0 & 0 & 0 & 1 & 1 & 1 & 1 \\
\hline
\end{tabular}

Abbreviations: EGFR, epidermal growth factor receptor; MoAb, monoclonal antibody.

*Thirteen patients received cetuximab as front-line therapy. 


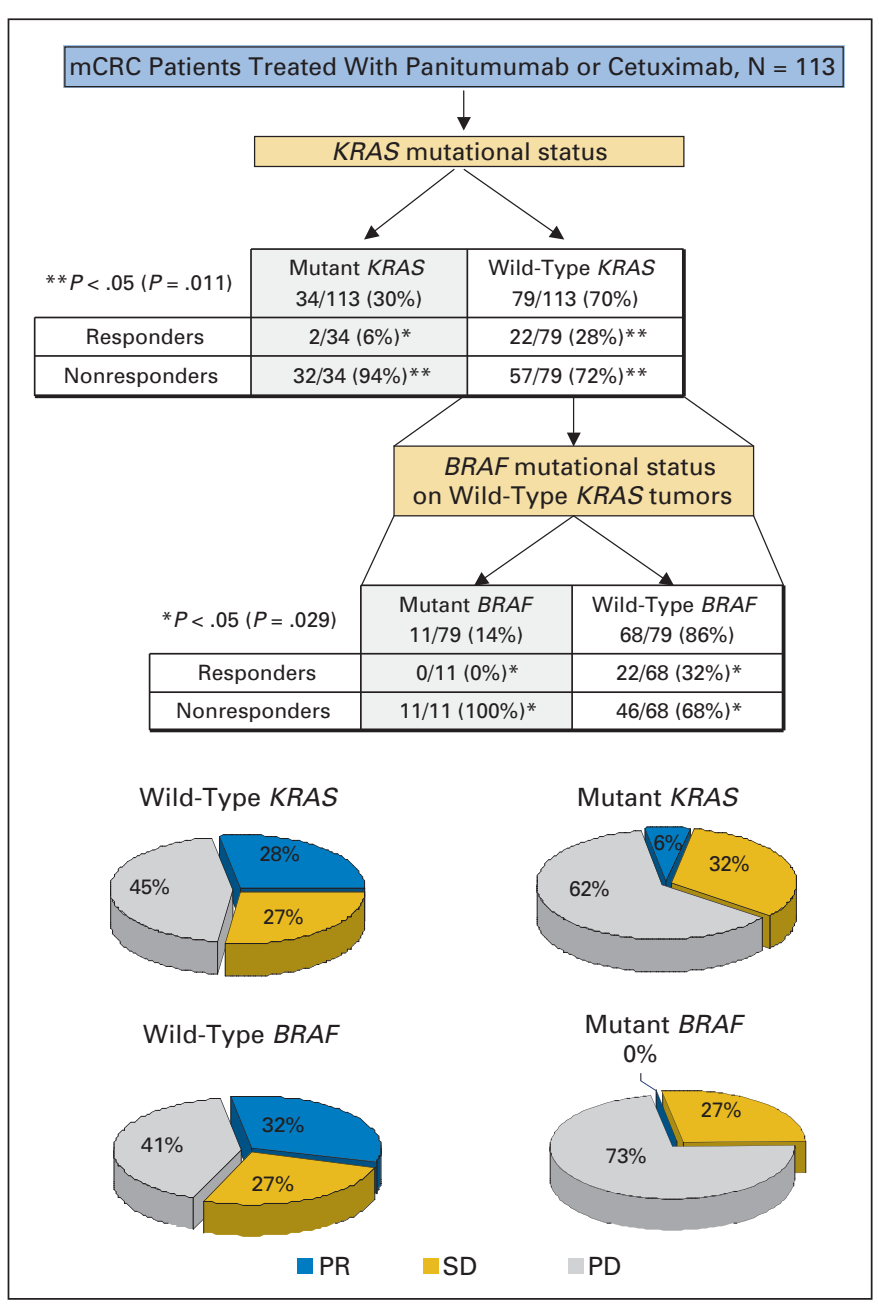

Fig 1. KRAS and BRAF mutations correlate with lack of response to treatment with monoclonal antibodies targeting epidermal growth factor receptor. The number of responders and nonresponders (stable disease [SD] + progressive disease [PD]) is indicated according to KRAS or BRAF mutational status. The percentage of patients displaying partial response (PR), SD, or PD is shown in the pie charts. $\mathrm{mCRC}$, metastatic colorectal cancer.

600 of $B R A F$, where the large majority of the mutations in these genes occur, as already reported. ${ }^{8,25}$ The list of primers used for mutational analysis is available from the authors on request. All samples were subjected to automated sequencing by ABI PRISM 3730 (Applied Biosystems, Foster City, CA). All mutated samples were confirmed twice, starting from independent polymerase chain reactions.

\section{Cellular Models, Cell Viability, Bromodeoxyuridine, and Apoptosis Assays}

Experimental procedures involving the use of cellular models have been described in the Appendix.

\section{Statistical Analysis}

The objective tumor response was the end point of our exploratory study. Qualitative comparisons of objective response to therapy (responders $[\mathrm{PR}] v$ nonresponders $[\mathrm{PD}+\mathrm{SD}]$ ) and gene mutations as predictors were performed by the two-tailed Fisher's exact test to check possible significance. The level of significance was set at $P<.05$. The progression-free survival (PFS) and overall survival (OS) analysis were determined according to the KaplanMeier method, and survival curves were compared using the log-rank test. PFS was defined as the time from start of treatment (see Patient Population and Treatment Regimens) until first documented tumor progression or death.
Data were analyzed using the Stata 9.1 package (Stata Corp, College Station, TX).

\section{RESULTS}

\section{Mutational Profiling of KRAS and Response of Patients Treated With Cetuximab or Panitumumab}

The mutational status of KRAS was assessed in a cohort of 113 patients ( 41 females and 72 males, with a mean age 63 years) who had received either panitumumab- or cetuximab-based chemotherapy. Twenty-four patients (21\%) achieved PR, and the median duration of response was 25 weeks (range, 10 to 128 weeks). Patient characteristics by KRAS mutational status are listed in Table 1. KRAS exon 2 mutations were found in $34(30 \%)$ of 113 analyzed patients. KRAS mutations were detected in two $(8 \%)$ of 24 responsive patients but in 32 (35\%) of 89 nonresponders (Fig 1). The data confirm that the presence of KRAS mutations correlates with lack of response to antiEGFR MoAb therapy (two-tailed Fisher's exact test, $P=.011$ ). The follow-up analyses indicated that patients carrying a KRASmutated tumor displayed a shorter PFS (log-rank test, $P=.0275$; Fig 2A). No statistically significant differences were observed by OS analysis $(P=.0869$; Fig $2 \mathrm{~B})$.

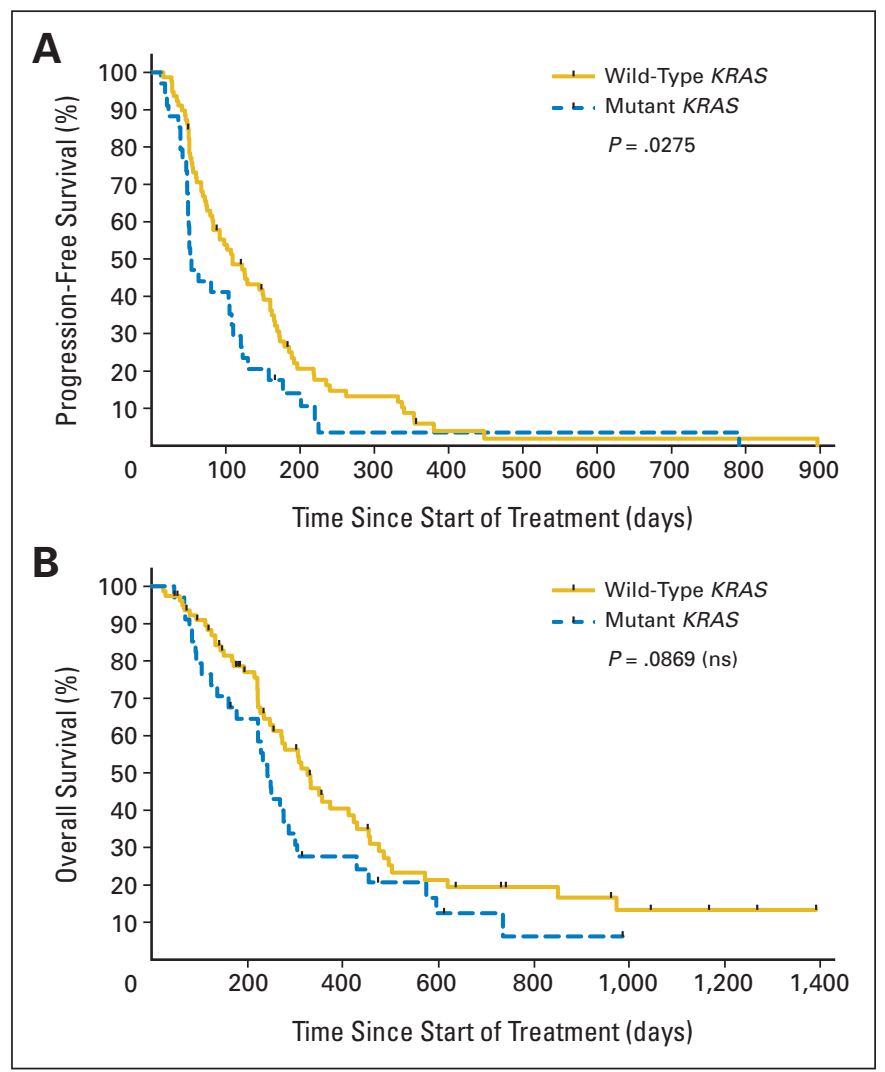

Fig 2. KRAS mutations are associated with shorter progression-free survival (PFS). (A) Patients with wild-type KRAS tumors displayed a better PFS than patients with KRAS-mutated tumors (log-rank test, $P=.0275)$. (B) Patients carrying KRAS-mutated tumors tended to have shorter overall survival. However, this difference did not reach statistical significance (log-rank test, $P=.0869$ ). ns, not significant. 

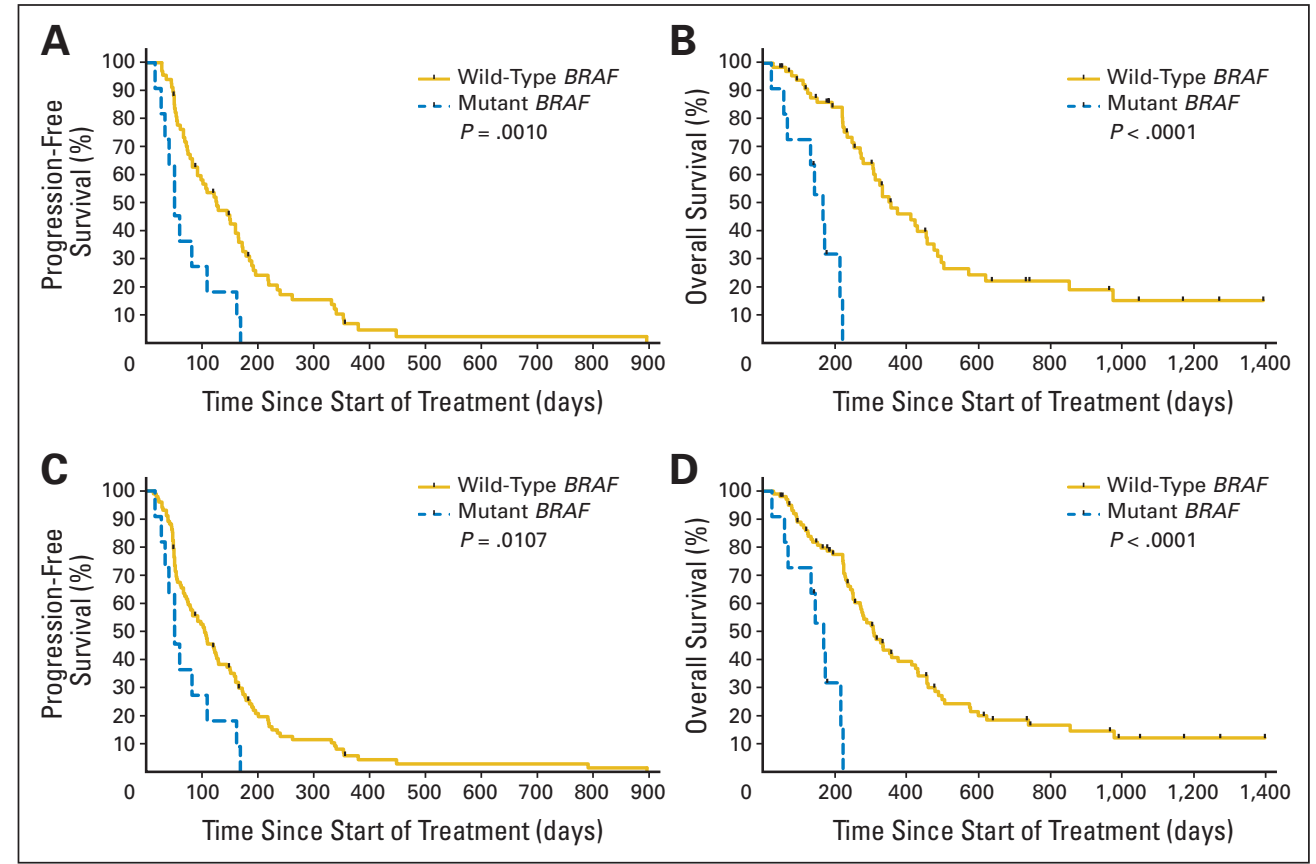

Fig 3. (A and B) In wild-type KRAS patients, those carrying a BRAF-mutated tumor had a shorter progression-free survival (PFS) and overall survival (OS) than wild-type BRAF patients (log-rank test, $P=.0010$ and $P<.0001$, respectively). ( $C$ and $D$ ) In the entire cohort of patients, individuals with wild-type $B R A F$ tumors still displayed longer PFS and OS than patients with BRAF-mutated tumors $(P=.0107$ and $P<.0001$, respectively).

\section{Mutational Profiling of BRAF in Wild-Type KRAS Patients Treated With Cetuximab or Panitumumab}

We and others have previously reported that KRAS and BRAF mutations are mutually exclusive in CRCs. ${ }^{26,27}$ This relationship was experimentally verified in our sample set, and we found that none of the KRAS-mutated samples carried concomitant BRAF mutations, or vice versa. The only type of $B R A F$ mutation found in our cohort was the previously described V600E substitution, which was detected in 11 patients $(13 \%)$. In all patients, the amino acid change was a result of the classic GTG $\rightarrow$ GAG mutation at position 1799 of the $B R A F$ nucleotide sequence. Patient characteristics by $B R A F$ mutational status are listed in Table 1.

\section{BRAF V600E Allele Impairs the Response to Cetuximab or Panitumumab}

The presence of $B R A F$ mutations was inversely associated with response to therapy (Fig 1). Significantly, none of 22 KRAS wild-type patients who experienced PR displayed $B R A F$ mutations, whereas 11 (14.0\%) of 79 nonresponder patients carried the BRAF V600E mutation (two-tailed Fisher's exact test, $P=.029$ ). These data indicate that occurrence of oncogenic BRAF alleles negatively interferes with the clinical response to MoAbs targeting EGFR.

\section{BRAF Mutations Are Associated With Shorter PFS and $O S$}

We assessed how the occurrence of BRAF mutations affected clinical parameters such as PFS and OS. The follow-up analyses indicated that within the subgroup of KRAS wild-type patients, those carrying a $B R A F$-mutated tumor had a shorter PFS and OS than BRAF wild-type patients (log-rank test, $P=.0010$ and $P<.0001$, respectively; Figs $3 \mathrm{~A}$ and $3 \mathrm{~B}$ ). In the entire cohort of patients, independently from KRAS mutational status, individuals with $B R A F$-mutated tumors still displayed shorter PFS and OS than patients with $B R A F$ wild-type tumors (log-rank test, $P=.0107$ and $P<.0001$, respec- tively; Figs 3C and 3D). This significant association indicates that the presence of $B R A F$ V600E mutation correlates with a worse prognosis. MSI has been linked both to the occurrence of BRAF mutation and to improved prognosis in sporadic CRC patients treated with fluorouracil-based regimens. ${ }^{19,28-30}$ MSI was assessed in a subset of patients, including 10 with BRAF-mutated samples, 24 with KRASmutated samples, and 41 with samples that were wild type for both genes. We identified MSI in one (1.3\%) of these 75 patients, and this patient had a sample that was wild type for both KRAS and BRAF. Importantly, none of the tested BRAF-mutated tumors displayed MSI, indicating that this factor is unlikely to affect the OS results and the effect on the efficacy of EGFR-targeted therapies presented earlier.

\section{BRAF V600E Allele Impairs the Response of CRC Cells to Cetuximab or Panitumumab}

The genetic profiling of CRCs showed that the presence of mutated BRAF negatively interferes with clinical response to EGFRtargeted MoAbs. We hypothesized that oncogenic activation of BRAF by the V600E mutation could bypass (short circuit) the EGFRinitiated signaling cascade. To assess the effect of the BRAF V600E mutation on response to cetuximab or panitumumab, we used cellular models of CRC. Specifically, we used a CRC line, DiFi, carrying amplification of the EGFR gene. ${ }^{8}$ We and others have previously shown that the proliferation of DiFi cells is inhibited by nanomolar concentration of cetuximab, and therefore, this line can be exploited to understand the molecular and cellular basis of sensitivity to antiEGFR MoAb therapy. ${ }^{8,31}$ We verified that DiFi cells do not show mutations in either KRAS or BRAF (data not shown), thus allowing further functional experiments. We found that DiFi cells expressing the mutated BRAF V600E allele (DiFi-BRAF) were less sensitive to either cetuximab or panitumumab compared with parental cells transduced with an empty vector (Figs $4 \mathrm{~A}$ and $4 \mathrm{~B}$ ). These data indicate that the presence of oncogenic BRAF (such as the V600E mutant) 


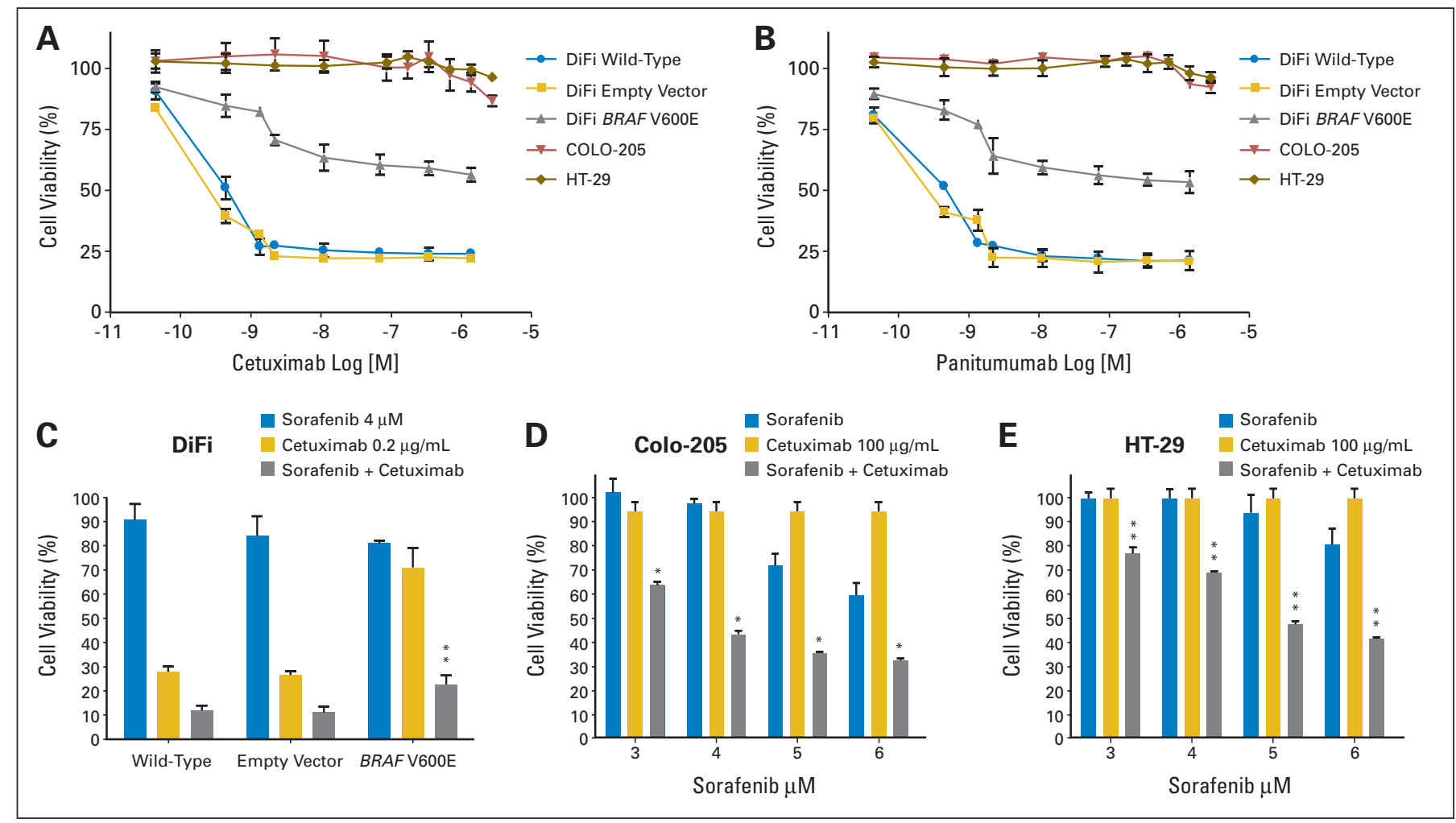

Fig 4. (A and B) The colorectal cancer cell line DiFi was transduced with either an empty vector or a BRAFV600E-encoding lentiviral vector. Cell viability was measured after treatment with either cetuximab or panitumumab. (C to E) Viability of DiFi, COLO-205, and HT-29 cells after combinatorial treatment with cetuximab plus sorafenib. Results (average \pm standard deviation) were normalized to untreated cells. $\left({ }^{*}\right) P<.05$ and $\left({ }^{*}\right) P<.01$ by Bonferroni multiple comparison test.

impairs the therapeutic potential of cetuximab and panitumumab in CRC cells.

To assess whether this finding could be replicated in cancer cells in which the BRAF V600E was naturally present, we used HT-29 and COLO-205, which are two cell lines derived from colorectal tumors in which the mutation had been previously detected. After independently verifying that both lines carried the BRAF V600E allele (and were wild type for KRAS), we found that HT-29 and COLO-205 are highly refractory to cetuximab and panitumumab treatment (Figs 4A and $4 \mathrm{~B})$.

\section{Combinatorial Targeting of EGFR and BRAF in CRC Cells Carrying the BRAF V600E Allele}

The mutational profiling of CRCs showed that the presence of $B R A F$ mutations renders cancer cells unresponsive to EGFR-targeted MoAb therapies. We hypothesized that the pharmacologic inhibition of $B R A F$ might restore the sensitivity to cetuximab or panitumumab in CRC cells carrying the V600E mutation. Sorafenib, a clinically approved small-molecule kinase inhibitor, was used to pharmacologically target $B R A F{ }^{32}$

When DiFi-BRAF, COLO-205, and HT-29 cells were treated with a combination of cetuximab and sorafenib, we found a dramatically reduced viability in all three cellular models (Figs 4C, 4D, and $4 \mathrm{E}$ ), whereas single agents, alone, had limited effects. To dissect the mechanism through which the combinatorial treatment synergistically affected cancer cells carrying the BRAF V600E mutation, we measured its effects both on cell cycle and on apoptosis. The combination of cetuximab and sorafenib limitedly affected proliferation compared with sorafenib alone (Fig 5A), whereas it showed a prominent proapoptotic effect, as demonstrated by the massive increase of caspase 3/7 activation observed in HT-29 and COLO205 cells (Fig 5B).

\section{DISCUSSION}

Cetuximab and panitumumab have shown efficacy in approximately $10 \%$ to $20 \%$ of mCRC patients. It is now emerging that genetic alterations of EGFR and its downstream signaling effectors may predict the efficacy of EGFR-targeted MoAbs. ${ }^{8-15}$ In particular, the role of oncogenic KRAS has been extensively analyzed, leading to the conclusion that the occurrence of KRAS mutations represents a predictive marker of anti-EGFR MoAb resistance. ${ }^{8-10,13-17}$ However, KRAS mutations only account for $30 \%$ to $40 \%$ of nonresponsive patients. Therefore, the identification of additional genetic determinants of primary resistance to EGFR-targeted therapies in CRC is clearly a priority. Genetic and biochemical evidence indicates that BRAF is the principal downstream effector of KRAS. ${ }^{33,34}$ Whether and to what extent the oncogenic activation of BRAF (a serine-threonine kinase mutated in approximately $10 \%$ of CRCs) affects the response to antiEGFR MoAbs has been only marginally investigated. ${ }^{13}$ By analyzing a larger cohort of mCRCs patients, we found that BRAF mutations are associated with resistance to EGFR-targeted MoAb therapies. Our data indicate that the role of $B R A F$ mutations in patients treated with EGFR-targeted drugs is similar to that played by mutated KRAS. We propose that the combined mutational analysis of both KRAS and 


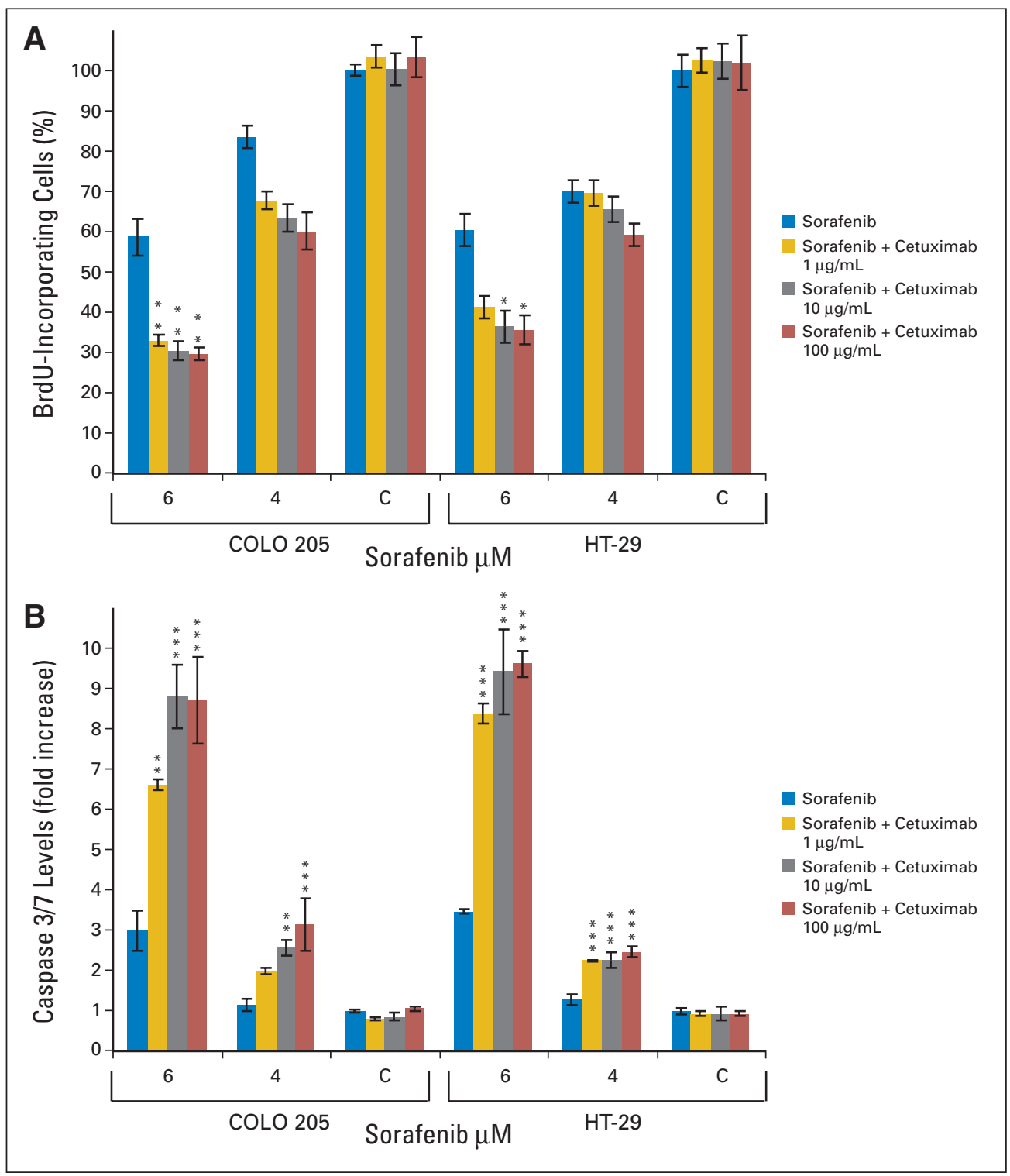

Fig 5. (A) Effects of combinatorial sorafenib plus cetuximab on proliferation by measuring bromodeoxyuridine (BrdU) incorporation in HT-29 and COLO-205 cells. (B) Levels of activated caspases 3/7 (apoptotic markers) after combinatorial treatment. Results (average \pm standard deviation) were normalized to untreated cells. $C$ indicates values for vehicle-treated (blue bars) or cetuximab-treated (yellow, gray, and red bars) cells. $\left(^{*}\right) P<.05$; $\left(^{* *}\right) P<.01$; and $(* *) P<.001$ by Bonferroni multiple comparison test.

$B R A F$ could be used to prospectively select mCRC patients eligible for EGFR-targeted MoAb treatment, with evident medical and economic implications. However, our findings need to be formally confirmed in a randomized clinical trial comparing an experimental arm containing cetuximab or panitumumab with a control arm without this targeted therapy.

Despite the predictive value of both KRAS and BRAF mutations, in our cohort, there was still a significant percentage of nonresponsive patients $(41 \%)$ who did not have mutations in either gene. Therefore, further molecular markers are needed to better define patients who are unlikely to benefit from EGFRtargeted MoAb treatment.

The present study also shows that patients carrying a BRAF point mutation have a shorter PFS and OS. This is in accordance with previous data indicating that CRCs with $B R A F$ mutations have a more aggressive phenotype. ${ }^{28}$ It has been previously reported that $B R A F$ mutations are more frequently detected in MSI-sporadic CRC compared with microsatellite-stable CRC (up to $50 \% v$ up to $12 \%$, respectively). ${ }^{28-30}$ Considering that MSI is a condition generally associated with better prognosis and with resistance to standard chemotherapies, ${ }^{19}$ we investigated this marker in our series. The tumors in which we detected BRAF mutations did not show MSI, thus excluding that the results could be a result of a general mechanism of MSIdependent drug resistance. Therefore, the prognostic and predictive value of $B R A F$ mutations in anti-EGFR-treated mCRC patients does not depend on the MSI status. The frequency of MSI in our cohort of advanced CRC patients is significantly lower (1.3\%) with respect to the expected rate (at least 15\%) when all stages of sporadic CRC are analyzed. We analyzed 30 additional mCRC patients (not treated with EGFR-targeted therapies), none of whom showed MSI. Therefore, our cumulative results indicate that only one of 105 advanced CRC patients showed MSI. A stage selection may account for these results because our cohort included only metastatic patients. In fact, our frequency is similar to that reported in a previous study that found only five patients (2.7\%) with MSI in 190 mCRC patients. ${ }^{35}$ Thus, our results are well in accordance with the notion that the MSI phenotype 
is associated with better prognosis ${ }^{19}$ and with a reduced likelihood of metastasis in patients with CRC. ${ }^{36}$

Although our study suggests a strong correlation of BRAF mutations in $\mathrm{mCRC}$ with a worse prognosis, further studies are needed to fully evaluate this marker with and without EGFR-targeted therapies, similar to what has been done for KRAS. ${ }^{18}$

To assess, at the molecular level, how the BRAF mutation could influence the response to anti-EGFR MoAbs, we exploited cellular models of CRC. We found that the occurrence or the exogenous introduction of the BRAF V600E allele in CRC cells dramatically impairs their response to cetuximab or panitumumab. These results have two relevant implications. First, they show that the presence of the V600E allele directly affects the response to EGFR-targeted MoAbs at the cellular level. Second, they suggest that the correlation between the occurrence of $B R A F$ mutation and the ineffective clinical response to cetuximab or panitumumab is not generically a result of the poorer prognosis of $B R A F$-mutated tumors. Our cell-based analysis also shows that even $B R A F$-mutated CRC cells can potentially respond to EGFR-targeted MoAbs if the BRAF inhibitor sorafenib is administered concomitantly with cetuximab or panitumumab. Of note, either drug alone had limited activity, indicating that they act synergistically. These data indicate that in BRAF-mutated tumors, the therapeutic effect of cetuximab or panitumumab could be restored by two-hit approaches aimed at blocking the EGFR pathway at multiple locations. In addition to sorafenib, other compounds targeting either BRAF (PLX4032) or its downstream effectors (ARRY-162, AZD6244, and PD0325901) are in clinical development ${ }^{37}$ and could be exploited in combination with EGFR-targeted MoAb therapy.

Overall, the present study has at least two implications that could be relevant for the therapy of $\mathrm{mCRC}$. The first is that patients whose tumors bear the BRAF V600E allele are not likely to experience significant clinical benefit on either cetuximab or panitumumab treatment. Therefore, BRAF mutation analysis could be used as an additional tool for the selection of mCRC patients who might benefit from EGFRtargeted MoAb therapies. The second is that clinical trials designed to test multiple therapies with EGFR and BRAF/MAPK inhibitors should be conceived for mCRC patients in whom the KRAS/BRAF pathway is oncogenically activated. Because cetuximab, panitumumab, and sorafenib are already approved for clinical use, the data presented here could find immediate clinical evaluation in phase II trials in patients who have BRAF-mutated tumors.

\section{AUTHORS' DISCLOSURES OF POTENTIAL CONFLICTS OF INTEREST}

The author(s) indicated no potential conflicts of interest.

\section{AUTHOR CONTRIBUTIONS}

Conception and design: Federica Di Nicolantonio, Miriam Martini, Francesca Molinari, Andrea Sartore-Bianchi, Piercarlo Saletti, Luca Mazzucchelli, Milo Frattini, Salvatore Siena, Alberto Bardelli

Financial support: Milo Frattini, Salvatore Siena, Alberto Bardelli Provision of study materials or patients: Federica Di Nicolantonio, Francesca Molinari, Andrea Sartore-Bianchi, Piercarlo Saletti, Sara De Dosso, Luca Mazzucchelli, Milo Frattini, Salvatore Siena,

Alberto Bardelli

Collection and assembly of data: Federica Di Nicolantonio, Miriam Martini, Francesca Molinari, Andrea Sartore-Bianchi, Sabrina Arena, Piercarlo Saletti, Sara De Dosso, Luca Mazzucchelli, Milo Frattini, Salvatore Siena, Alberto Bardelli

Data analysis and interpretation: Federica Di Nicolantonio, Miriam Martini, Francesca Molinari, Andrea Sartore-Bianchi, Piercarlo Saletti, Sara De Dosso, Luca Mazzucchelli, Milo Frattini, Salvatore Siena, Alberto Bardelli

Manuscript writing: Federica Di Nicolantonio, Francesca Molinari, Andrea Sartore-Bianchi, Piercarlo Saletti, Luca Mazzucchelli, Milo Frattini, Salvatore Siena, Alberto Bardelli

Final approval of manuscript: Federica Di Nicolantonio, Miriam Martini, Francesca Molinari, Andrea Sartore-Bianchi, Piercarlo Saletti, Sara De Dosso, Luca Mazzucchelli, Milo Frattini, Salvatore Siena, Alberto Bardelli

\section{REFERENCES}

1. Venook AP: Epidermal growth factor receptortargeted treatment for advanced colorectal carcinoma. Cancer 103:2435-2446, 2005

2. Saltz LB, Meropol NJ, Loehrer PJ Sr, et al: Phase II trial of cetuximab in patients with refractory colorectal cancer that expresses the epidermal growth factor receptor. J Clin Oncol 22:1201-1208, 2004

3. Cunningham $D$, Humblet $Y$, Siena $S$, et al: Cetuximab monotherapy and cetuximab plus irinotecan in irinotecan-refractory metastatic colorectal cancer. N Engl J Med 351:337-345, 2004

4. Meyerhardt JA, Mayer RJ: Systemic therapy for colorectal cancer. N Engl J Med 352:476-487, 2005

5. Van Cutsem E, Peeters $M$, Siena $S$, et al: Open-label phase III trial of panitumumab plus best supportive care compared with best supportive care alone in patients with chemotherapy-refractory metastatic colorectal cancer. J Clin Oncol 25:1658-1664, 2007

6. Baselga J: The EGFR as a target for anticancer therapy: Focus on cetuximab. Eur J Cancer 37:S16S22, 2001 (suppl 4)

7. Chung KY, Shia J, Kemeny NE, et al: Cetuximab shows activity in colorectal cancer patients with tumors that do not express the epidermal growth factor receptor by immunohistochemistry. J Clin Oncol 23:1803-1810, 2005

8. Moroni $M$, Veronese $S$, Benvenuti $S$, et al: Gene copy number for epidermal growth factor receptor (EGFR) and clinical response to antiEGFR treatment in colorectal cancer: A cohort study. Lancet Oncol 6:279-286, 2005

9. Lièvre $A$, Bachet JB, Le Corre $D$, et al: KRAS mutation status is predictive of response to cetuximab therapy in colorectal cancer. Cancer Res 66: 3992-3995, 2006

10. Frattini $M$, Saletti $P$, Romagnani $E$, et al: PTEN loss of expression predicts cetuximab efficacy in metastatic colorectal cancer patients. $\mathrm{Br} \mathrm{J}$ Cancer 97:1139-1145, 2007

11. Sartore-Bianchi A, Moroni M, Veronese $S$, et al: Epidermal growth factor receptor gene copy number and clinical outcome of metastatic colorectal cancer treated with panitumumab. J Clin Oncol 25:3238-3245, 2007

12. Cappuzzo F, Finocchiaro G, Rossi E, et al: EGFR FISH assay predicts for response to cetuximab in chemotherapy refractory colorectal cancer patients. Ann Oncol 19:717-723, 2008

13. Benvenuti $S$, Sartore-Bianchi A, Di Nicolantonio $F$, et al: Oncogenic activation of the RAS/RAF signaling pathway impairs the response of metastatic colorectal cancers to anti-epidermal growth factor receptor antibody therapies. Cancer Res 67: 2643-2648, 2007

14. Di Fiore $F$, Blanchard $F$, Charbonnier $F$, et al: Clinical relevance of KRAS mutation detection in metastatic colorectal cancer treated by cetuximab plus chemotherapy. $\mathrm{Br} J$ Cancer 96:1166-1169, 2007

15. De Roock W, Piessevaux H, De Schutter J, et al: KRAS wild-type state predicts survival and is associated to early radiological response in metastatic colorectal cancer treated with cetuximab. Ann Oncol 19:508-515, 2008

16. Khambata-Ford S, Garrett CR, Meropol NJ, et al: Expression of epiregulin and amphiregulin and K-ras mutation status predict disease control in metastatic colorectal cancer patients treated with cetuximab. J Clin Oncol 25:3230-3237, 2007

17. Lièvre $A$, Bachet JB, Boige $V$, et al: KRAS mutations as an independent prognostic factor in patients with advanced colorectal cancer treated with cetuximab. J Clin Oncol 26:374-379, 2008

18. Amado RG, Wolf $M$, Peeters $M$, et al: Wildtype KRAS is required for panitumumab efficacy in patients with metastatic colorectal cancer. J Clin Oncol 26:1626-1634, 2008 
19. Locker GY, Hamilton S, Harris J, et al: ASCO 2006 update of recommendations for the use of tumor markers in gastrointestinal cancer. J Clin Oncol 24:5313-5327, 2006

20. Borner M, Koeberle D, Von Moos R, et al: Adding cetuximab to capecitabine plus oxaliplatin (XELOX) in first-line treatment of metastatic colorectal cancer: A randomized phase II trial of the Swiss Group for Clinical Cancer Research SAKK. Ann Oncol 19:1288-1292, 2008

21. Pessino A, Artale $S$, Sciallero $S$, et al: First-line single-agent cetuximab in patients with advanced colorectal cancer. Ann Oncol 19:711-716, 2008

22. Van Cutsem E, Siena S, Humblet $Y$, et al: An open-label, single-arm study assessing safety and efficacy of panitumumab in patients with metastatic colorectal cancer refractory to standard chemotherapy. Ann Oncol 19:92-98, 2008

23. Wilke $H$, Glynne-Jones $R$, Thaler J, et al: MABEL: A large multinational study of cetuximab plus irinotecan in pretreated metastatic colorectal cancer progressing on irinotecan. J Clin Oncol [epub ahead of print on October 14, 2008]

24. Therasse $P$, Arbuck SG, Eisenhauer EA, et al: New guidelines to evaluate the response to treatment in solid tumors: European Organization for Research and Treatment of Cancer, National Cancer
Institute of the United States, National Cancer Institute of Canada. J Natl Cancer Inst 92:205-216, 2000

25. Frattini M, Ferrario $C$, Bressan $P$, et al: Alternative mutations of BRAF, RET and NTRK1 are associated with similar but distinct gene expression patterns in papillary thyroid cancer. Oncogene 23: 7436-7440, 2004

26. Frattini M, Balestra D, Suardi S, et al: Different genetic features associated with colon and rectal carcinogenesis. Clin Cancer Res 10:4015-4021, 2004

27. Rajagopalan $H$, Bardelli $A$, Lengauer $C$, et al: Tumorigenesis: RAF/RAS oncogenes and mismatchrepair status. Nature 418:934, 2002

28. Samowitz WS, Sweeney C, Herrick J, et al: Poor survival associated with the BRAF V600E mutation in microsatellite-stable colon cancers. Cancer Res 65:6063-6069, 2005

29. Deng G, Bell I, Crawley S, et al: BRAF mutation is frequently present in sporadic colorectal cancer with methylated hMLH1, but not in hereditary nonpolyposis colorectal cancer. Clin Cancer Res 10:191-195, 2004

30. Wang L, Cunningham JM, Winters JL, et al: BRAF mutations in colon cancer are not likely attributable to defective DNA mismatch repair. Cancer Res 63:5209-5212, 2003
31. Lu Y, Li $X$, Liang $K$, et al: Epidermal growth factor receptor (EGFR) ubiquitination as a mechanism of acquired resistance escaping treatment by the anti-EGFR monoclonal antibody cetuximab. Cancer Res 67:8240-8247, 2007

32. Wilhelm SM, Carter $C$, Tang $L$, et al: BAY 43-9006 exhibits broad spectrum oral antitumor activity and targets the RAF/MEK/ERK pathway and receptor tyrosine kinases involved in tumor progression and angiogenesis. Cancer Res 64:7099-7109, 2004

33. Yan J, Roy S, Apolloni A, et al: Ras isoforms vary in their ability to activate Raf-1 and phosphoinositide 3-kinase. J Biol Chem 273:24052-24056, 1998

34. Zhang BH, Guan KL: Activation of B-Raf kinase requires phosphorylation of the conserved residues Thr598 and Ser601. Embo J 19:5429-5439, 2000

35. Haddad R, Ogilvie RT, Croitoru M, et al: Microsatellite instability as a prognostic factor in resected colorectal cancer liver metastases. Ann Surg Oncol 11:977-982, 2004

36. Malesci A, Laghi $L$, Bianchi $P$, et al: Reduced likelihood of metastases in patients with microsatelliteunstable colorectal cancer. Clin Cancer Res 13:38313839, 2007

37. Friday BB, Adjei AA: Advances in targeting the Ras/Raf/MEK/Erk mitogen-activated protein kinase cascade with MEK inhibitors for cancer therapy. Clin Cancer Res 14:342-346, 2008

\section{Acknowledgment}

We thank Chris Torrance, Luca Cardone, and Fonnet Bleeker for critically reading the manuscript; Simona Lamba for assistance with sequencing; Silvio Veronese and Marcello Gambacorta for pathology assessments; Salvatore Artale for clinical care; and Giovanna Marrapese and Ines Andreotti for data management.

\section{Glossary Terms}

BRAF V600E: The V600E is the most common oncogenic mutation of BRAF in cancer. The V600E aminoacid change results in constitutive activation of the BRAF kinase and promotes cell transformation.

PI3K-PTEN-AKT pathway: Signal transduction pathways involving the signaling molecules phosphatidylinositol-3 kinase (PI3K), PTEN and Akt. PI3K generates phosphorylated inositides at the cell membrane which are required for the recruitment and activation of the serine kinase Akt. PTEN is a lipid phosphatase which counteracts the effect of PI3K. Accordingly mutated PI3K and AKT act as dominant oncogenes while PTEN is a tumor suppressor gene.

Cetuximab: Also called Erbitux or C225, cetuximab is a monoclonal antibody that is designed to target EGFR and block its signaling activity by initiating receptor activation.

Panitumumab: Also known as ABX-EGF, panitumumab is a fully human immunoglobulin G2 monoclonal antibody against EGFR and inhibits ligand-induced activation of the EGFR. This antibody is generated from mouse strains engineered to be deficient in mouse antibody production and to produce fully human antibodies.

KRAS: The gene that encodes K-Ras, a protein that is a member of the small GTPase superfamily, in which a single amino acid substitution results in an activating mutation. Alternative splicing gives rise to variants encoding two isoforms that differ in the C-terminal region.
BRAF: BRAF is an isoform of RAF. Raf proteins (Raf-1, A-Raf, B-Raf) are intermediate to Ras and MAPK in the cellular proliferative pathway. Raf proteins are typically activated by Ras via phosphorylation, and activated Raf proteins in turn activate MAPK via phosphorylation. However, Raf proteins may also be independently activated by other kinases.

Sorafenib: A substance belonging to the family of drugs called raf kinase inhibitors and anti-VEGF that is being studied in the treatment of cancer.

MAPK (mitogen-activated protein kinase): MAPKs are a family of enzymes that form an integrated network influencing cellular functions such as differentiation, proliferation, and cell death. These cytoplasmic proteins modulate the activities of other intracellular proteins by adding phosphate groups to their serine/threonine amino acids.

MSI (microsatellite instability): Microsatellites are repeating units of 1-4 DNA base pairs that are distributed widely throughout the genome and have a high degree of repeat length variation in the population. Their length remains stable with cell division and inheritance so they may be used as molecular markers of cell lineage, in population genetic studies or paternity testing. Defects in the genes involved in DNA mismatch repair result in genomic instability that may be detected as MSI, an alteration in the length of the microsatellites from cell to cell. 